\title{
Creation of an Ultracold Neutral Plasma
}

\author{
Scott D. Bergeson \\ scott.bergeson@byu.edu \\ T. C. Killian \\ S. Kulin \\ L. A. Orozco \\ C. Orzel
}

See next page for additional authors

Follow this and additional works at: https://scholarsarchive.byu.edu/facpub

Part of the Astrophysics and Astronomy Commons, and the Physics Commons

\section{Original Publication Citation}

T. Killian, S. Kulin, S. D. Bergeson, L. Orozco, and S. L. Rolston, "Creation of an ultracold neutral plasma," Physical Review Letters, 83, 4776-4779 (1999). The original publication of this article can be found at http://link.aps.org/doi/1.113/PhysRevLett.83.4776

\section{BYU ScholarsArchive Citation}

Bergeson, Scott D.; Killian, T. C.; Kulin, S.; Orozco, L. A.; Orzel, C.; and Rolston, S. L., "Creation of an Ultracold Neutral Plasma" (1999). Faculty Publications. 607.

https://scholarsarchive.byu.edu/facpub/607

This Peer-Reviewed Article is brought to you for free and open access by BYU ScholarsArchive. It has been accepted for inclusion in Faculty Publications by an authorized administrator of BYU ScholarsArchive. For more information, please contact ellen_amatangelo@byu.edu. 
Authors

Scott D. Bergeson, T. C. Killian, S. Kulin, L. A. Orozco, C. Orzel, and S. L. Rolston 


\title{
Creation of an Ultracold Neutral Plasma
}

\author{
T. C. Killian, S. Kulin, S. D. Bergeson, ${ }^{*}$ L. A. Orozco, ${ }^{\dagger}$ C. Orzel, and S. L. Rolston \\ National Institute of Standards and Technology, Gaithersburg, Maryland 20899-8424
}

(Received 30 July 1999)

\begin{abstract}
We report the creation of an ultracold neutral plasma by photoionization of laser-cooled xenon atoms. The charge carrier density is as high as $2 \times 10^{9} \mathrm{~cm}^{-3}$, and the temperatures of electrons and ions are as low as $100 \mathrm{mK}$ and $10 \mu \mathrm{K}$, respectively. Plasma behavior is evident in the trapping of electrons by the positive ion cloud when the Debye screening length becomes smaller than the size of the sample. We produce plasmas with parameters such that both electrons and ions are strongly coupled.
\end{abstract}

PACS numbers: 52.55.Dy, 32.80.Pj, 52.25.Ub, 52.50.Jm

The study of ionized gases in neutral plasma physics spans temperatures ranging from $10^{16} \mathrm{~K}$ in the magnetosphere of a pulsar to $300 \mathrm{~K}$ in the earth's ionosphere [1]. At lower temperatures, the properties of plasmas are expected to differ significantly. For instance, three-body recombination, which is prevalent in high temperature plasmas, should be suppressed [2]. If the thermal energy of the particles is less than the Coulomb interaction energy, the plasma becomes strongly coupled, and the usual hydrodynamic equations of motion and collective mode dispersion relations are no longer valid [3]. Strongly coupled plasmas are difficult to produce in the laboratory and only a handful of examples exist [4], but such plasmas do occur naturally in astrophysical systems.

In this work, we create an ultracold neutral plasma with an electron temperature as low as $T_{e}=100 \mathrm{mK}$, an ion temperature as low as $T_{i}=10 \mu \mathrm{K}$, and densities as high as $n=2 \times 10^{9} \mathrm{~cm}^{-3}$. We obtain this novel plasma by photoionization of laser-cooled xenon atoms. Within the experimentally accessible ranges of temperatures and densities, both components can be simultaneously strongly coupled. A simple model describes the evolution of the plasma in terms of the competition between the kinetic energy of the electrons and the Coulomb attraction between electrons and ions. A numerical calculation accurately reproduces the data.

Photoionization and laser cooling have been used before in plasma experiments. Photoionization in a $600 \mathrm{~K}$ Cs vapor cell produced a plasma with $T_{e} \geq 2000 \mathrm{~K}$ [5], and a strongly coupled non-neutral plasma was created by laser cooling magnetically trapped $\mathrm{Be}^{+}$ions [6].

A plasma is often defined as an ionized gas in which the charged particles exhibit collective effects [7]. The length scale which divides individual particle behavior and collective behavior is the Debye screening length $\lambda_{D}$. It is the distance over which an electric field is screened by redistribution of electrons in the plasma, and is given by $\lambda_{D}=\sqrt{\epsilon_{0} k_{B} T_{e} / e^{2} n}$. Here, $\epsilon_{0}$ is the electric permittivity of vacuum, $k_{B}$ is the Boltzmann constant, and $e$ is the elementary charge. An ionized gas is not a plasma unless the Debye length is smaller than the size of the system [7]. In our experiment, the Debye length can be as low as $500 \mathrm{~nm}$, while the size of the sample is $\sigma \approx 200 \mu \mathrm{m}$. The condition $\lambda_{D}<\sigma$ for creating a plasma is thus easily fulfilled.

The atomic system we use is metastable xenon in the $6 s[3 / 2]_{2}$ state. This state has a lifetime of $43 \mathrm{~s}$ [8] and can be treated as the ground state for laser cooling on the transition at $882 \mathrm{~nm}$ to the $6 p[5 / 2]_{3}$ state [9]. The metastable atoms are produced in a discharge and subsequently decelerated using the Zeeman slowing technique. The atoms are then collected in a magneto-optical trap and further cooled with optical molasses to approximately $10 \mu \mathrm{K}$. We characterize the cold neutral atoms by optical absorption imaging [10]. This measurement provides the density and size of the atomic cloud and the number of atoms in the sample. Typically we prepare a few million atoms at a density of $\approx 2 \times 10^{10}$ atoms $/ \mathrm{cm}^{3}$. Their spatial distribution is Gaussian with a rms radius $\sigma \approx 200 \mu \mathrm{m}$.

We partially ionize the cold atom sample via two photon excitation. A pulse of light from the cooling laser at $882 \mathrm{~nm}$ populates the $6 p[5 / 2]_{3}$ level. Green photons $(\lambda=514 \mathrm{~nm})$ from a pulsed dye laser, pumped by a frequency-tripled pulsed Nd:YAG laser, then excite atoms to states at or above the ionization potential.

The energy difference, $\Delta E$, between the photon energy and the ionization potential is distributed between the electrons and ions. Because of the large ion to electron mass ratio, all except $4 \times 10^{-6} \Delta E$ is given to the electrons. Equipartition of energy between ions and electrons requires tens of ms [11]. We vary $\Delta E / k_{B}$ in a controlled manner between $0.1-1000 \mathrm{~K}$ by changing the green laser frequency. The bandwidth of the laser of $0.07 \mathrm{~cm}^{-1}$ sets the lower limit.

By adjusting the pulse energy of the green laser, we control the number of atoms photoionized. For the highest energy available, $1 \mathrm{~mJ}$ in a $10 \mathrm{~ns}$ pulse, we can produce up to $2 \times 10^{5}$ ions, which corresponds to a peak density of $2 \times 10^{9} \mathrm{~cm}^{-3}$. The number of atoms photoionized varies linearly with the laser intensity. Although the ionization fraction is low $(\leq 10 \%)$, the charged particles show no evidence of interactions with the neutral atoms. This is to be expected because the mean free path for 
neutral-charged particle collisions is much greater than the sample size [12-14].

For detection of charged particles, an external electric field is applied to direct ions towards a microchannel plate detector and electrons towards a single channel electron multiplier. The efficiencies are $50 \%$ for ions [15] and $85 \%$ for electrons. The magnitude of the applied electric field is calibrated through field ionization of Rydberg atoms [16].

In each cycle of the experiment, the atoms are first laser cooled and an electric field of approximately $0.005 \mathrm{~V} / \mathrm{cm}$ is applied. The atoms are then photoionized, and after about $500 \mathrm{~ns}$ of time of flight a pulse of electrons arrives at the detector (see Fig. 1). If the green laser energy is high enough, the first peak develops a tail, and a second peak appears when the electric field is linearly increased a few microseconds later. On this time scale the ions are essentially stationary. About $300 \mu$ s after the electric field ramp is applied, they are detected on the microchannel plates.

A simple model (Fig. 2) explains the experimental data. The charge distribution is everywhere neutral immediately after photoionization. Because of the initial kinetic energy of the electrons $(\approx \Delta E)$, the electron cloud begins expanding. The resulting local charge imbalance creates an internal electric field which produces a Coulomb potential energy well for electrons. If the well never becomes deeper than the initial kinetic energy, all the electrons escape. This corresponds to the uppermost curve (i.e., lowest laser intensity) in Fig. 1. If enough atoms are photoionized, however, only an outer shell of electrons escapes, and the well becomes deep enough to trap the rest. Electrons in the well redistribute their energy through collisions within 10-100 ns [11]. As charges are promoted to energies above the trap depth, they leave the well. This

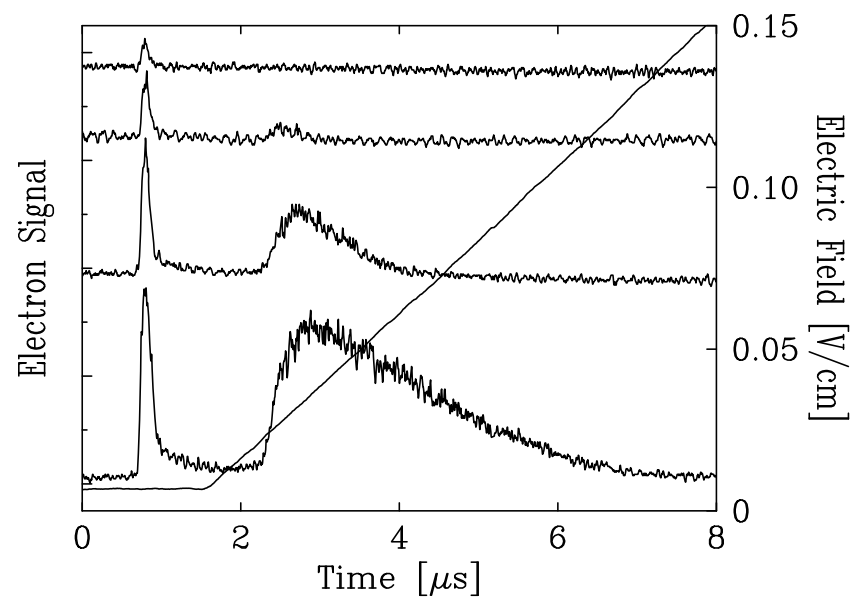

FIG. 1. Electron signals recorded for four different pulse energies of the green laser, i.e., different densities of charged particles $\left(10^{5}-10^{7} \mathrm{~cm}^{-3}\right)$. The uppermost curve corresponds to the lowest energy. The photoionization occurs at $t=0$. The initial kinetic energy of the electrons is $\Delta E / k_{B}=0.6 \mathrm{~K}$. The data shown are an average over 20 cycles of the experiment. Also shown is the magnitude of the applied electric field. explains the tail of the first peak in the electron signal. During this process of evaporation the potential well depth increases. Evaporation eventually slows and remaining electrons are held until an applied electric field overcomes the trapping potential. They appear as the second peak in Fig. 1.

This description suggests that for a given $\Delta E$ there is a threshold number of positive ions required for trapping electrons. The data show such behavior in a plot of the fraction of electrons trapped versus the number of photoions produced (Fig. 3a). As $\Delta E$ increases, more positive charges are required to produce the trapping effect.

At the trapping threshold, after all the electrons have left, the potential well depth equals the initial kinetic energy of the electrons. From this relation one can calculate the number of positive ions at the threshold, $N^{*}=\Delta E / U_{0}$. Here, $U_{0}=\sqrt{2 / \pi} e^{2} / 4 \pi \varepsilon_{0} \sigma$, and $\sigma$ is the rms radius of the Gaussian spatial distribution of positive ions.

The data agree with this simple calculation. For a wide range of electron kinetic energies, the onset of trapping occurs at $N=N^{*}$, as shown in Fig. 3b. Scaling the number of ions produced by $N^{*}$ shows that all data fall on a universal curve [17]. A numerical simulation which approximates the initial velocity, $v=\sqrt{2 \Delta E / m}$, as directed radially outward and integrates the equations of motion for the electrons, reproduces this behavior.

The model described above (Fig. 2) implies that the temperature of the electrons is $T_{e} \lesssim \Delta E / k_{B}$. This is

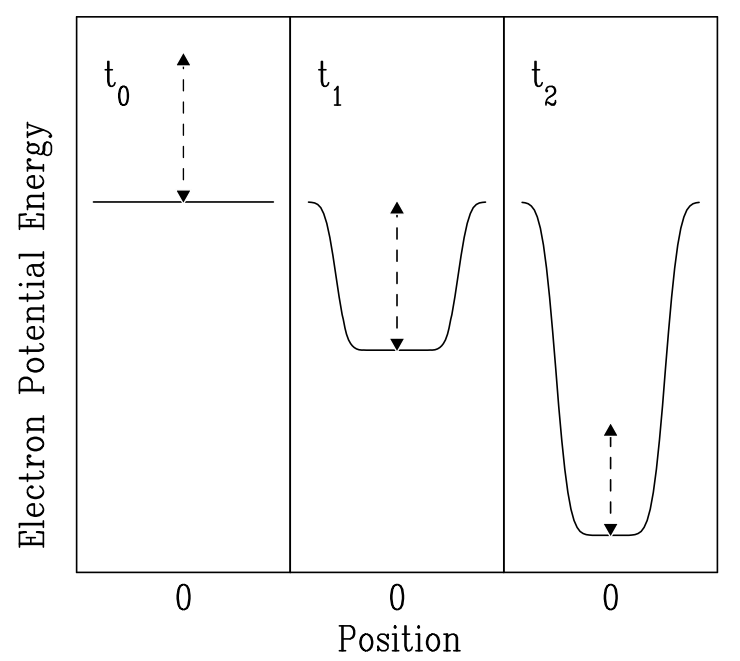

FIG. 2. Schematic of the potential energy seen by a test electron when enough atoms are photoionized to result in trapping of electrons. Photoionization occurs at $t_{0}=0$ and the sample is everywhere neutral. Because of the kinetic energy imparted by the laser, some electrons leave and a charge imbalance develops. At $t_{1} \approx 10 \mathrm{~ns}$ the resulting potential well equals the initial kinetic energy, trapping the remaining electrons. Because of Debye screening, the bottom of the well is flat. As electrons in the well thermalize, evaporation occurs. The well depth increases and the electrons cool slightly. By $t_{2} \approx 1 \mu \mathrm{s}$ evaporation essentially stops. The dashed line indicates the average kinetic energy of the electrons. 


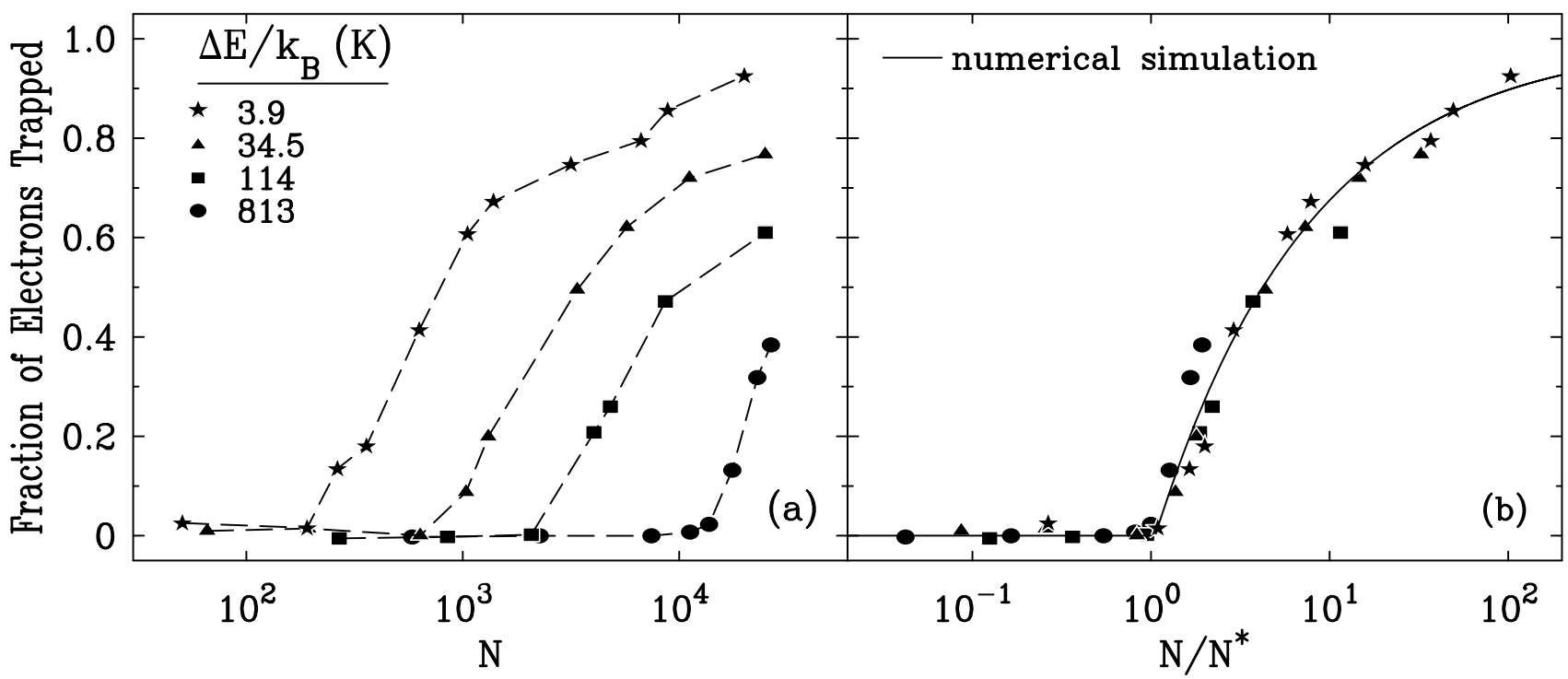

FIG. 3. (a) The fraction of electrons trapped is plotted versus the number of photoions created. Each curve corresponds to a different green laser frequency. The corresponding initial energies of the electrons are displayed in the legend. As the energy increases, more positive ions are required to trap electrons. (b) Same as (a) but the number of photoions is scaled by $N^{*}$. The threshold for trapping is given by $N=N^{*}$. The line is the result of a numerical simulation. There is a scale uncertainty of about $10 \%$ in determining the fraction of electrons trapped.

confirmed by the numerical simulation. In principle, the energy distribution of the trapped electrons can also be determined from the shape of the second peak in Fig. 1. However, the analysis is complicated because the trap depth increases as electrons are removed. Also, rethermalization is fast and the temperature changes on the time scale of the electric field ramp.

The initial ion temperature is easily estimated. For excitation close to the ionization threshold, the energy imparted to the ions from photoionization is negligible compared to the kinetic energy of the atoms. Therefore the minimum initial temperature is $10 \mu \mathrm{K}$. For large $\Delta E$ the temperature approaches $4 \times 10^{-6} \Delta E / k_{B}$, which is $4 \mathrm{mK}$ for $\Delta E=1000 \mathrm{~K}$. Although the equilibration time is on the order of tens of ms, collisions with the energetic electrons are expected to approximately double the ion temperature within a $\mu \mathrm{s}$.

The threshold condition $N=N^{*}$ is mathematically equivalent to $\lambda_{D}=\sigma$. In this context, one can interpret $\lambda_{D}$ as the displacement of electrons from their equilibrium positions when their energy in the local internal electric field in the plasma equals their kinetic energy [7]. If $\lambda_{D}>\sigma$, the electrons are free to escape to infinity. If $\lambda_{D}<\sigma$, electrons are trapped in the ion cloud by the internal field and a plasma is formed.

After the untrapped fraction of electrons has escaped, the cloud as a whole is no longer strictly neutral. But, as mentioned above, electrons escape most easily from the edges of the spatial distribution, and for $N>N^{*}$ the center of the cloud is well described as a neutral plasma. This behavior is also seen in the numerical simulation.

The only significant effect of the residual charge imbalance is a Coulomb expansion of the cloud that occurs on a long time scale of many microseconds. This limits the time available for studying the highest density conditions. The expansion also decreases the potential well depth, allowing formerly trapped electrons to escape. In a plasma with 5000 ions and $10 \%$ charge imbalance, half of the initially trapped electrons escape in about $100 \mu \mathrm{s}$. The expansion is slowed compared to what would be observed for a bare cloud of positive charges, however. A bare cloud of 5000 ions, initially with $\sigma=200 \mu \mathrm{m}$, expands to twice its radius in a few microseconds, reducing the well depth by a factor of 2 .

Phenomena similar to the electron trapping observed here are seen in traditional plasmas. For instance, recombination can often occur at containment walls and leads to net charge diffusion from the center of the plasma. The mobility of the electrons is larger than that of the ions, but their motion is retarded by local internal electric fields which develop from any charge imbalance. This leads to ambipolar diffusion [1] in which electrons and ions migrate at equal rates.

In our ultracold plasma the thermal energy of the charged particles can be less than the Coulomb interaction energy between nearest neighbors, making it strongly coupled. The situation is characterized quantitatively by the electron and ion Coulomb coupling parameters [18] $\Gamma_{e}=\left(e^{2} / 4 \pi \varepsilon_{0} a\right) / k_{B} T_{e}$ and $\Gamma_{i}=\mathrm{e}^{-a / \lambda_{D}} \Gamma_{e} T_{e} / T_{i}$ where $a=(4 \pi n / 3)^{-1 / 3}$ is the Wigner-Seitz radius. The exponential term in the expression for $\Gamma_{i}$ is due to the shielding of the ion-ion interaction by electrons [19]. When $\Gamma>1$, many-body spatial correlations [20] exist and phase transitions such as crystallization [21] of the sample may occur. Systems with $\Gamma_{e}>1$ are sometimes called non-Debye plasmas because $\lambda_{D}<a$. For the 
densities and temperatures accessed in the experiment, we can prepare a plasma in which both electrons and ions are initially strongly coupled: $\Gamma_{e}=10$ and $\Gamma_{i}=1000$. To our knowledge such a system has never been created before.

This novel plasma is well suited for a wide range of experiments. Plasma oscillations [7,22], which have a frequency $f_{p}=\sqrt{n e^{2} / m \epsilon_{0}} / 2 \pi$ of up to $400 \mathrm{MHz}$, can be used to probe the density distribution of the system. Magnetic confinement may greatly extend the plasma lifetime, and, because of the low sample temperature, the required field should be small. The thermalization and evaporative cooling of electrons, and the temperature of the ions, require further study.

We can also look for three-body electron-ion recombination. At higher temperatures, the rate for this process scales as $T^{-9 / 2}$ [23], and an extrapolation to the present experimental conditions yields a recombination time of nanoseconds (for $T_{e}=1 \mathrm{~K}$ and $n=2 \times 10^{8} \mathrm{~cm}^{-3}$ ). The long lifetime we observe $(\sim 100 \mu \mathrm{s})$ is the first clear indication that this theory, as well as an extension to $T \approx 1 \mathrm{~K}$ [2], breaks down for the temperatures studied here.

The initial kinetic energy of the electrons can be reduced to $\approx 10 \mathrm{mK}$ by using a laser with a bandwidth equal to the Fourier transform limit of a $10 \mathrm{~ns}$ pulse. One may be able to decrease this energy even further by exciting below the ionization limit. In this case, one creates a dense gas of highly excited cold Rydberg atoms for which many-body interactions can cause a phase transition to a plasmalike state [24]. In preliminary experiments we have observed the formation of free electrons and ions in such a system. This will be the subject of future experiments.

The technique to produce ultracold plasmas demonstrated in this work is applicable to any atom that can be laser cooled, and other atoms may offer experimental advantages. With alkali systems, one can attain higher initial densities, and alkaline earth ions have accessible optical transitions.

In summary, we have accessed a new region in the parameter space of neutral plasmas by photoionizing a cloud of laser-cooled atoms. Conditions were realized in which both electrons and ions are strongly coupled. Experimentally, the initial plasma properties are easily controlled and the evolution of the system is described with an uncomplicated model.

S. Kulin acknowledges funding from the Alexandervon-Humboldt foundation, and T.C. Killian is supported by NRC. This work was supported by ONR. *Present address: Department of Physics and Astronomy,
Brigham Young University, Provo, Utah 84602-4640.
†Present address: Department of Physics and Astronomy,
State University of New York, Stony Brook, New York
11794-3800.

[1] J.-L. Delcroix and A. Bers, Physique des Plasmas (InterEditions/CNRS Editions, Paris, 1994), Vol. 1.

[2] Y. Hahn, Phys. Lett. A 231, 82 (1997).

[3] G. Kalman, K. I. Golden, and M. Minella, in Strongly Coupled Plasma Physics, edited by H. M. Van Horn and S. Ichimaru (University of Rochester, Rochester, 1993), p. 323.

[4] For a review, see Strongly Coupled Plasma Physics, edited by H. M. Van Horn and S. Ichimaru (University of Rochester, Rochester, 1993).

[5] O. L. Landen and R. J. Winfield, Phys. Rev. Lett. 54, 1660 (1985).

[6] T.B. Mitchell, J. J. Bollinger, D.H.E. Dubin, X.-P. Huang, W. M. Itano, and R. H. Baughman, Science 282, 1290 (1998); W. M. Itano, J. J. Bollinger, J. N. Tan, B. Jelenković, X.-P. Huang, and D. J. Wineland, Science 279, 686 (1998).

[7] F. F. Chen, Introduction to Plasma Physics (Plenum, New York, 1974).

[8] M. Walhout, A. Witte, and S. L. Rolston, Phys. Rev. Lett. 72, 2843 (1994).

[9] M. Walhout, H. J. L. Megens, A. Witte, and S. L. Rolston, Phys. Rev. A 48, R879 (1993).

[10] M. Walhout, U. Sterr, C. Orzel, M. Hoogerland, and S. L. Rolston, Phys. Rev. Lett 74, 506 (1995).

[11] L. Spitzer, Jr., Physics of Fully Ionized Gases (Wiley, New York, 1962).

[12] R. Haberland, L. Fritsche, and J. Noffke, Phys. Rev. A 33, 2305 (1986).

[13] B. Plenkiewicz, P. Plenkiewicz, C. Houée-Levin, and J.-P. Jay-Gerin, Phys. Rev. A 38, 6120 (1988).

[14] T. J. McIlrath and T. B. Lucatorto, Phys. Rev. Lett. 38, 1390 (1977).

[15] J. Oberheide, P. Wilhelms, and M. Zimmer, Meas. Sci. Technol. 8, 351 (1997).

[16] T.F. Gallagher, Rydberg Atoms (Cambridge University, Cambridge, England, 1994).

[17] For electron kinetic energies $\Delta E / k_{B} \leq 1 \mathrm{~K}$, the data are not well described by the simple model because the trapping behavior is dominated by the extraction field and unknown stray electric fields. The presence of an external electric field modifies the threshold condition. The expression for $N^{*}$ becomes $N^{*} \approx \frac{1}{U_{0}}\{\Delta E+2 e F \sigma[1+$ $\sqrt{1+(\Delta E / e F \sigma)}]\}$, where $F$ is the magnitude of the external electric field.

[18] S. Ichimaru, Rev. Mod. Phys. 54, 1017 (1982).

[19] G. E. Morfill, H. M. Thomas, U. Konopka, and M. Zuzic, Phys. Plasmas 6, 1769 (1999).

[20] D. H. E. Dubin and T. M. O’Neil, Rev. Mod. Phys. 71, 87 (1999).

[21] For a review, see the special issue on Invited and Tutorial Papers from the 40th Annual Meeting of the Division of Plasma Physics of the APS [Phys. Plasmas 6, 1751 (1999)].

[22] L. Tonks and I. Langmuir, Phys. Rev. 33, 195 (1929).

[23] P. Mansbach and J. Keck, Phys. Rev. 181, 275 (1969).

[24] G. Vitrant, J. M. Raimond, M. Gross, and S. Haroche, J. Phys. B, At. Mol. Phys. 15, L49 (1982). 